International Journal of Applied Mathematical Research, 1 (4) (2012) 643-658

(C) Science Publishing Corporation

www.sciencepubco.com/index.php/IJAMR

\title{
Fuzzy Linear Fractional Bi-Level Multi-Objective Programming Problems
}

\author{
M. saraj, N. safaei \\ Department of Mathematics, Faculty of Mathematical Sciences \\ and Computer, Shahid Chamran University, Ahvaz- Iran \\ Email: msaraj@scu.ac.ir \\ Email: n_safaei@ymail.com
}

\begin{abstract}
The Kuhn-Tuker condition has become nowadays an important tool in the hands of investigation for checking the optimality in optimization literature. In the present paper with use of a Taylor series and KuhnTucker conditions approach, we solve a fuzzy linear fractional bilevel multi-objective programming (FLFBL-MOP) problem. The Taylor series is an expansion of a series that represents a function. In the proposed approach, membership functions associated with each level(s) of the objective(s) of FLFBL-MOP problems are transformed and unified by using a Taylor series approach. By using the Kuhn-Tucker conditions, the problem is reduced to a single objective and finally, numerical example is given to illustrates the efficiency and superiority of the proposed approach.
\end{abstract}

Keywords: Fuzzy programming, fractional programming, bi-level multiobjective programming, Kuhn-Tucker conditions, Taylor series.

\section{Introduction}

Bi-level mathematical programming (BLMP) is identified as mathematical programming that solves decentralized planning problems with two decision makers (DMs) in a two level or hierarchical organization. The basic concept of the BLMP technique is that the upper level decision maker (ULDM) (the leader) sets his goals and/or decisions and then asks each subordinate level of 
the organization for their optima which are calculated in isolation; the lower level DM (LLDM) (the follower) decisions are then submitted and modified by the ULDM with consideration of the overall benefit for the organization; the process continued until a satisfactory solution is reached. In other words, although the ULDM independently optimizes its own benefits, the decision may be affected by the reaction of the LLDM. As a consequence, decision deadlock arises frequently and the problem of distribution of proper decision power is encountered in most of the practical decision situations. Most of the developments on BLMP problems focus on bi-level linear programming [1-4], and many others for bilevel nonlinear programming and bi-level multiobjective programming $[3,5,6]$. The use of the fuzzy set theory for decision problems with several conflicting objectives was first introduced by Zimmermann[7]. There after, various versions of fuzzy programming have been investigated and widely circulated in literature. In a hierarchical decision making context, it has been realized that each DM should have a motivation to cooperate with other, and a minimum level of satisfaction of the DM at a lower-level must be considered for overall benefit of the organization. The use of the concept of membership function of fuzzy set theory to BLMP problems for satisfactory decisions was first introduced by Lai[8]. The basic concept of the fuzzy programming approaches implies that the LLDM optimizes his/her objective function, taking a goal or preference of the ULDM into consideration. In the decision process, considering the membership functions of the fuzzy goals for the decision variables of the ULDM, the LLDM solves a FP problem with the set of constraints on an overall satisfactory degree of the ULDM. If the proposed solution is not satisfactory to the ULDM, the solution search is continued by redefining the elicited membership functions until a satisfactory solution is reached[9]. The main difficulty that arises with the FP approach of Shih et al [10], is that there is possibility of rejecting the solution again and again by the ULDM and reevaluation of the problem is repeatedly needed to reach the satisfactory decision, where the objectives of the DMs are overconflicting. Even inconsistency between the fuzzy goals of the objectives and the decision variables may arise. This makes the solution process a lengthy one [9]. Fuzzy programming approach to multi-level programming problems was studied by Sinha [11]. The Baky investigated the problem of Fuzzy goal programming algorithm for solving decentralized bi-level multi-objective programming problems[12]. In this paper, membership functions, which are associated with each objectives of each levels of FLFBL-MOP. are transformed to linear form by using first-order Taylor polynomial series. Here, the obtained Taylor series which has polynomial membership functions are equivalent to fractional membership functions which is associated to each objectives of each levels and Reduce the FLFBL-MOP into a single objective.

In other words, suitable transformation can be applied to formulate an 
equivalent fuzzy linear fractional bileve multi objective programming problem. The performance of the proposed method was experimentally validated by example. Results demonstrate that the proposed approach runs more effectively.

\section{Problem Formulation}

Assume that there are two levels in a hierarchy structure with upper level decision maker(ULDM)and lower level decision maker(LLDM). Let the vector of decision variables $x=\left(x_{1}, x_{2}\right)$ be partitioned between the two planners. The upper level decision maker has control over the vector $x_{1} \in R^{n_{1}}$ and the lower level decision maker has control over the vector $x_{2} \in R^{n_{2}}$, where $n=n_{1}+n_{2}$. Furthermore, assume that

$$
F_{i}\left(x_{1}, x_{2}\right): R^{n_{1}} \times R^{n_{2}} \longrightarrow R^{m_{i}}, \quad i=1,2
$$

are the upper level and lower level vector objective functions respectively. So the LFBL-MOP problem of maximization type may be formulated as follows $[5,12]$ :

[1st level]

$$
\operatorname{Max}_{x_{1}} F_{1}\left(x_{1}, x_{2}\right)=\operatorname{Max}\left(f_{11}\left(x_{1}, x_{2}\right), f_{12}\left(x_{1}, x_{2}\right), \ldots, f_{1 m_{1}}\left(x_{1}, x_{2}\right)\right)
$$

where $x_{2}$ solves

[2nd level]

$$
\begin{aligned}
& \underset{\text { Max }}{\operatorname{Subject}} F_{2}\left(x_{1}, x_{2}\right)=\operatorname{Max}\left(f_{21}\left(x_{1}, x_{2}\right), f_{22}\left(x_{1}, x_{2}\right), \ldots, f_{2 m_{2}}\left(x_{1}, x_{2}\right)\right) \\
& x \in G=\left\{x \in R^{n} \mid A_{1} x_{1}+A_{2} x_{2} \leqslant b, x \geqslant 0, b \in R^{m}\right\} \neq \varnothing
\end{aligned}
$$

Where

$$
f_{i j}\left(x_{1}, x_{2}\right)=\frac{c_{i j} x+\alpha_{i j}}{d_{i j} x+\beta_{i j}}
$$

for $\mathrm{i}=1$, we have $\mathrm{j}=1,2, \ldots, m_{1}$, for ULDM objective functions, for $\mathrm{i}=2$, we have $\mathrm{j}=1,2, \ldots, m_{2}$, for LLDM objective functions, and where

(1) $x_{1} \in R^{n_{1}}, x_{2} \in R^{n_{2}}$,

(2) $\mathrm{G}$ is the bi-level convex constraints feasible choice set,

(3) $m_{1}$ is the number of first-level objective functions,

(4) $m_{2}$ is the number of second-level objective functions,

(5) $\mathrm{m}$ is the number of the constraints,

(6) $A_{i}: m \times n_{i}$ matrix, $\mathrm{i}=1,2$, 
(7) $c_{i j}, d_{i j} \in R^{n}, d_{i j} x+\beta_{i j}>0$ for all $x \in G$,

(8) $\alpha_{i j}, \beta_{i j}$ are constants.

\section{Fuzzy Linear Fractional Bilevel Multi-Objective Programming}

If an imprecise aspiration level is introduced to each of the level of BFP, then we call it as a fuzzy level. The FLFBL-MOP can be written as follows:

Find $x_{1}$

soastosatisfy

$f_{1 j}\left(x_{1}, x_{2}\right) \lesssim g_{1 j} \quad\left(f_{1 j}\left(x_{1}, x_{2}\right) \gtrsim g_{1 j}\right), \mathrm{j}=1,2, \ldots, m_{1}$

where $x_{2}$ solves

$f_{2 j}\left(x_{1}, x_{2}\right) \lesssim g_{2 j} \quad\left(f_{2 j}\left(x_{1}, x_{2}\right) \gtrsim g_{2 j}\right), j=1,2, \ldots, m_{2}$

subjectto

$$
\begin{aligned}
& A x_{1}+A x_{2} \leqslant b \\
& x_{1}, x_{2} \geqslant 0, x_{1} \in R^{n_{1}}, x_{2} \in R^{n_{2}}, A_{1} \in R^{m \times n_{1}}, \\
& A_{2} \in R^{m \times n_{2}}, b \in R^{m}
\end{aligned}
$$

where $g_{1 j}$ is the aspiration level of the ULDM and $g_{2 j}$ is the aspiration level of the LLDM. $\lesssim$ and $\gtrsim$ indicate fuzziness of the aspiration levels, which are described as essentially less than and essentially more than, respectively. $\lesssim$ minimizes $f_{i j}\left(x_{1}, x_{2}\right)$ and $\gtrsim$ maximizes $f_{i j}\left(x_{1}, x_{2}\right)$.

A membership function must be described for each fuzzy level. A membership function can be ststed as follows for $\mathrm{i}=1,2$ and $\mathrm{j}=1, \ldots, m_{i}$ :

if $f_{i j}\left(x_{1}, x_{2}\right) \gtrsim g_{i j}$

$$
\mu_{f_{i j}}\left(f_{i j}(x)\right)=\left\{\begin{array}{cc}
1 & f_{i j}\left(x_{1}, x_{2}\right) \geqslant g_{i j} \\
\frac{f_{i j}\left(x_{1}, x_{2}\right)-\underline{t_{i j}}}{g_{i j}-\underline{t_{i j}}} \underline{\underline{t_{i j}}} \leqslant f_{i j}\left(x_{1}, x_{2}\right) \leqslant g_{i j} \\
0 & f_{i j}\left(x_{1}, x_{2}\right) \leqslant \underline{t_{i j}}
\end{array}\right.
$$

if $f_{i j}\left(x_{1}, x_{2}\right) \lesssim g_{i j}$

$$
\mu_{f_{i j}}\left(f_{i j}(x)\right)=\left\{\begin{array}{cc}
1 & f_{i j}\left(x_{1}, x_{2}\right) \leqslant g_{i j} \\
\frac{\overline{t_{i j}}-f_{i j}\left(x_{1}, x_{2}\right)}{\overline{t_{i j}}-g_{i j}} & g_{i j} \leqslant f_{i j}\left(x_{1}, x_{2}\right) \leqslant \overline{t_{i j}} \\
0 & f_{i j}\left(x_{1}, x_{2}\right) \geqslant \overline{t_{i j}}
\end{array}\right.
$$

where $\overline{t_{i j}}$ and $t_{i j}$ are the upper and lower tolerance limits, respectively, for each fuzzy level. 


\section{The Taylor Series and Kuhn-Tucker Condi- tions for Solving Flfbl-Mop Problems}

In the FLFBL-MOLP problems, membership functions associated to each of the objectives in each level are firstly transformed by using Taylor series and then a satisfactory value(s) for the variable(s) of the model is obtained by solving the fuzzy model, which has a single objective function. Here, the fractional linear membership functions from each objectives of each levels is converted to a linear polynomial on using Taylor series . Then, the FLFBLMOLP on using Kuhn-Tucker conditions can be reduced to a single objective. The proposed approach can be explained in four steps.

Step 1. Determine $x_{i j}^{*}=\left(x_{i j}^{1 *}, x_{i j}^{2 *}\right)\left(\mathrm{i}=1,2\right.$ and $\left.\mathrm{j}=1,2, . . ., m_{i}\right)$ which is the value(s) that is used to maximize the each of the objectives in upper level and lower level membership Function $\mu_{f_{i j}}(x)$ associated to upper level and lower level $f_{i j}\left(x_{1}, x_{2}\right)$ ( $\mathrm{i}=1,2$ and $\left.\mathrm{j}=1,2, . . ., m_{i}\right)$ where $\mathrm{n}$ is the number of the variables.

Step 2. Transform membership functions by using first-order Taylor polynomial series

$$
\begin{gathered}
\mu_{f_{i j}}\left(f_{i j}(x)\right) \cong \widehat{\mu_{f_{i j}}}\left(f_{i j}(x)\right)=\mu_{f_{i j}}\left(f_{i j}\left(x_{i j}^{*}\right)\right)+\left(\left(x_{1}-x_{i j}^{1 *}\right) \frac{\partial}{\partial x_{1}}+\right. \\
\left.\left(x_{2}-x_{i j}^{2 *}\right) \frac{\partial}{\partial x_{2}}\right) \mu_{f_{i j}}\left(f_{i j}\left(x_{i j}^{*}\right)\right) \\
\mu_{f_{i j}}\left(f_{i j}(x)\right) \cong \widehat{\mu_{f_{i j}}}\left(f_{i j}(x)\right)=\mu_{f_{i j}} f_{i j}\left(x_{i j}^{*}\right)+\sum_{k=1}^{2}\left(x_{k}-x_{i j}^{k *}\right) \frac{\left.\partial \mu_{f_{i j}} f_{i j}\left(x_{i j}^{*}\right)\right)}{\partial x_{k}}
\end{gathered}
$$

Step 3. Sum the Membership functions together for the upper level. Note that problem is solved by assuming that weights of the objectives in upper level are equal.

$$
P(x)=\sum_{j=1}^{m_{1}}\left(\mu_{f_{1 j}}\left(f_{1 j}\left(x_{1 j}^{*}\right)\right)+\sum_{k=1}^{2}\left(x_{k}-x_{1 j}^{k *}\right) \frac{\partial \mu_{f_{1 j}}\left(f_{1 j}\left(x_{1 j}^{*}\right)\right)}{\partial x_{k}}\right)
$$

Step 4. After applying the Kuhn-Tucker conditions to the lower level of the objective problem, we find satisfactory $x^{*}=\left(x^{*}{ }_{1}, x_{2}^{*}\right)$ by solving the reduced problem to a single objective.

FLFBP is converted into a new mathematical model. This model is represent as follows: 


$$
\begin{aligned}
& \text { Max } P(x) \\
& \text { s.t } \\
& A_{1} x_{1}+A_{2} x_{2}+u=b \\
& w A_{2}-\nu=\sum_{j=1}^{m_{2}} \frac{\partial \mu_{f_{2 j}}\left(f_{2 j}\left(x_{2 j}^{*}\right)\right)}{\partial x_{2}} \\
& w u=0, x_{2} \nu=0 \\
& x_{1}, x_{2}, w, u, \nu \geqslant 0
\end{aligned}
$$

In this method, a zero-one variable, $\eta$ and $\xi$, is added for each constraint $w u=0$ and $x_{2} \nu=0$, respectively. In addition, each of these constraints is replaced by two linear inequalities involving $\eta$ and $\xi$ and M, a large positive. The auxiliary formulation now becomes

$$
\begin{array}{ll}
\operatorname{Max} & P(x) \\
\text { s.t } & A_{1} x_{1}+A_{2} x_{2}+u=b \\
& w A_{2}-\nu=\sum_{j=1}^{m_{2}} \frac{\partial \mu_{f_{2 j}}\left(f_{2 j}\left(x_{2 j}^{*}\right)\right)}{\partial x_{2}} \\
& w \leqslant M \eta, u \leqslant M(1-\eta) \\
& x_{2} \leqslant M \xi, \nu \leqslant M(1-\xi) \\
& \eta, \quad \xi \in\{0,1\} \\
& x_{1}, \quad x_{2}, \quad w, u, \nu \geqslant 0
\end{array}
$$

\section{Numerical Example}

An illusrative numerical example.

$$
\operatorname{Max}_{x_{1}}\left(f_{11}=\frac{2 x_{1}+x_{2}+2}{2 x_{2}+1}, f_{12}=\frac{x_{1}}{x_{1}+x_{2}+1}\right)
$$

where $x_{2}$ solve

$$
\operatorname{Max}\left(f_{21}=\frac{3 x_{1}+2 x_{2}-1}{-x_{1}+x_{2}+4}, f_{22}=\frac{-x_{1}+x_{2}+3}{x_{1}+5}\right)
$$

subject to

$$
\begin{aligned}
& x_{1}+x_{2} \leqslant 4 \\
& x_{1}-2 x_{2} \leqslant 1 \\
& 3 x_{1}+x_{2} \geqslant 3 \\
& x_{1}, x_{2} \geqslant 0
\end{aligned}
$$


Let the fuzzy aspiration levels of the objectives in the bilevel to be $(3,0$, $1,1)$, respectively, so we have:

Find $x_{1}$

soastosatisfy

where $x_{2}$ solve

$$
f_{11}=\frac{2 x_{1}+x_{2}+2}{2 x_{2}+1} \gtrsim 3, \quad f_{12}=\frac{x_{1}}{x_{1}+x_{2}+1} \gtrsim 0
$$

$$
f_{21}=\frac{3 x_{1}+2 x_{2}-1}{-x_{1}+x_{2}+4} \gtrsim 1, \quad f_{22}=\frac{-x_{1}+x_{2}+3}{x_{1}+5} \gtrsim 1
$$

subjectto

$$
\begin{aligned}
& x_{1}+x_{2} \leqslant 4 \\
& x_{1}-2 x_{2} \leqslant 1 \\
& 3 x_{1}+x_{2} \geqslant 3 \\
& x_{1}, x_{2} \geqslant 0
\end{aligned}
$$

Let us assume that, the tolerance limits of the objectives in the bilevel are $(1,1,1,1)$ respectively. The membership functions of the bilevel are as follows:

First, membership functions are defined to be simple piecewise linear (see Fig.1). The membership functions of the levels are obtained as follows:

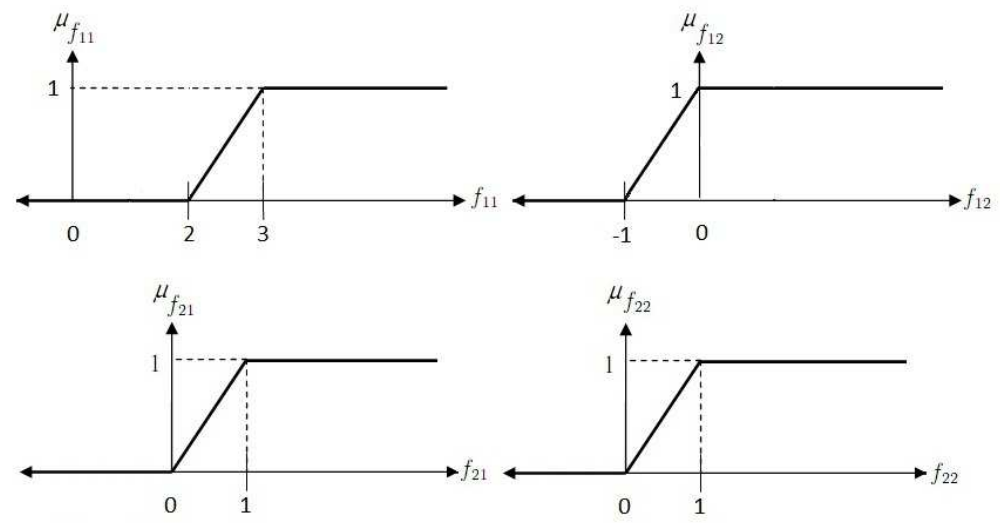

Figure 1: simple piecewise linear membership functions.

$$
\begin{aligned}
& \mu_{f_{11}}\left(f_{11}(x)\right)=\left\{\begin{array}{cc}
1 & \text { if } f_{11}\left(x_{1}, x_{2}\right) \geqslant g_{11} \\
\frac{f_{11}\left(x_{1}, x_{2}\right)-\underline{t_{11}}}{g_{11}-\underline{t_{11}}} & \text { if } \underline{t_{11}} \leqslant f_{11}\left(x_{1}, x_{2}\right) \leqslant g_{11} \\
0 & \text { if } f_{11}\left(x_{1}, x_{2}\right) \leqslant \underline{t_{11}}
\end{array}\right. \\
&=\left\{\begin{array}{cll}
1 & \text { if } f_{11}\left(x_{1}, x_{2}\right) \geqslant 3 \\
\frac{2 x_{1}-3 x_{2}}{2 x_{2}+1} & \text { if } 2 \leqslant f_{11}\left(x_{1}, x_{2}\right) \leqslant 3 \\
0 & \text { if } f_{11}\left(x_{1}, x_{2}\right) \leqslant 2
\end{array}\right.
\end{aligned}
$$


In the same way, the other membership functions are formed as

$$
\begin{aligned}
& \mu_{f_{12}}\left(f_{12}(x)\right)=\left\{\begin{array}{cl}
1 & \text { if } f_{12}\left(x_{1}, x_{2}\right) \geqslant 0 \\
\frac{2 x_{1}+x_{2}+1}{x_{1}+x_{2}+1} & \text { if }-1 \leqslant f_{12}\left(x_{1}, x_{2}\right) \leqslant 0 \\
0 & \text { if } f_{12}\left(x_{1}, x_{2}\right) \leqslant-1
\end{array}\right. \\
& \mu_{f_{21}}\left(f_{21}(x)\right)=\left\{\begin{array}{cl}
1 & \text { if } f_{21}\left(x_{1}, x_{2}\right) \geqslant 1 \\
\frac{3 x_{1}+2 x_{2}-1}{-x_{1}+x_{2}+4} & \text { if } 0 \leqslant f_{21}\left(x_{1}, x_{2}\right) \leqslant 1 \\
0 & \text { if } f_{21}\left(x_{1}, x_{2}\right) \leqslant 0
\end{array}\right. \\
& \mu_{f_{22}}\left(f_{22}(x)\right)=\left\{\begin{array}{cll}
\frac{-x_{1}+x_{2}+3}{x_{1}+5} & \text { if } f_{22}\left(x_{1}, x_{2}\right) \geqslant 1 \\
0 & \text { if } ~ & f_{22}\left(x_{1}, x_{2}\right) \leqslant 0
\end{array}\right.
\end{aligned}
$$

If the problem is solved for each membership functions, one by one, then $\mu_{f_{11}}^{*}\left(f_{11}(1,0)\right), \mu_{f_{12}}^{*}\left(f_{12}(3,1)\right), \mu_{f_{21}}^{*}\left(f_{21}(3,1)\right)$ and $\mu_{f_{22}}^{*}\left(f_{22}(0,4)\right)$ are obtained. Now the membership functions are transformed by using first-order Taylor polynomial series.

$$
\begin{aligned}
\mu_{f_{11}}\left(f_{11}(x)\right) \cong \widehat{\mu_{f_{11}}}\left(f_{11}(x)\right)=\mu_{f_{11}}\left(f_{11}(1,0)\right)+\left(\left(x_{1}-1\right) \times \frac{\partial}{\partial x_{1}}\right. \\
\\
\left.+\left(x_{2}-0\right) \times \frac{\partial}{\partial x_{2}}\right) \mu_{f_{11}}\left(f_{11}(1,0)\right)=2 x_{1}-5 x_{2}
\end{aligned}
$$

In the same manner, the other membership functions are transformed on using first-order Taylor polynomial series as follows:

$$
\begin{aligned}
& \mu_{f_{12}}\left(f_{12}(x)\right)=1.52+.08 x_{1}-0.12 x_{2} \\
& \mu_{f_{21}}\left(f_{21}(x)\right)=5.5+4 x_{1}-1.5 x_{2} \\
& \mu_{f_{22}}\left(f_{22}(x)\right)=0.6-0.48 x_{1}+0.2 x_{2}
\end{aligned}
$$

The $\mathrm{P}(\mathrm{x})$ is obtained by adding (14) and (15) as follows:

$$
P(x)=\mu_{f_{11}}\left(f_{11}(x)\right)+\mu_{f_{12}}\left(f_{12}(x)\right)=1.52+2.08 x_{1}-5.12 x_{2}
$$

After applying the Kuhn-Tucker conditions to the lower level of the objectives problem, a new auxiliary problem is to be solved 


$$
\begin{array}{cl}
\operatorname{Max} & \quad P(x)=1.52+2.08 x_{1}-5.12 x_{2} \\
\text { s.t } & \\
& x_{1}+x_{2}+u_{1}=4 \\
& x_{1}-2 x_{2}+u_{2}=1 \\
& 3 x_{1}+x_{2}-u_{3}=3 \\
& w_{1}-2 w_{2}-w_{3}-\nu=-1.5+0.2 \\
& w_{i} \leqslant M \eta_{i}, \quad u_{i} \leqslant M\left(1-\eta_{i}\right) \\
& x_{2} \leqslant M \xi, \quad \nu \leqslant M(1-\xi) \\
& \eta_{i}, \quad \xi \in\{0,1\}, \\
& x_{1}, \quad x_{2}, w_{i}, \quad u_{i}, \quad \nu \geqslant 0 \quad i=1, \ldots, 3
\end{array}
$$

We solve the problem for $\mathrm{M}=1000$ and the solution is obtained as follows:

$$
f_{11}\left(x_{1}, x_{2}\right)=4, f_{12}\left(x_{1}, x_{2}\right)_{1}^{x_{1}^{*}=1}=0.5, \quad f_{21}\left(x_{1}^{*}, x_{2}\right)=0.67, f_{22}\left(x_{1}, x_{2}\right)=0.33
$$

and the membership values are

$$
\mu_{f_{11}}\left(f_{11}\right)=1, \quad \mu_{f_{12}}\left(f_{12}\right)=1, \quad \mu_{f_{21}}\left(f_{21}\right)=0.67, \quad \mu_{f_{22}}\left(f_{11}\right)=0.33
$$

The membership function values indicates that the levels $f_{11} \& f_{12}$ are satisfied $100 \%$ but $f_{21}$ is satisfied $67 \%$ and $f_{22}$ is satisfied $33 \%$ with the solutions of $x_{1}^{*}=1$ and $x_{2}^{*}=0$.

Now further more we assume that the membership functions to be triangular (see Fig.2) which depends on three scalar parameters (a, b, c). Let $f_{11}$ be depends on three scalar parameters $(3,4,5)$ where $f_{12}$ depends on $(0$, $0.5,1), f_{21}$ on $(0,1,3)$ and $f_{22}$ be depends on $(-1,1,3)$. As shown given by $f_{11}\left(x_{1}, x_{2} ; 3,4,5\right), f_{12}\left(x_{1}, x_{2} ;(0,0.5,1), f_{21}\left(x_{1}, x_{2} ;(0,1,3)\right.\right.$ and $f_{22}\left(x_{1}, x_{2} ;(-1,1,3)\right.$.

The membership functions of the level are obtained as follows:
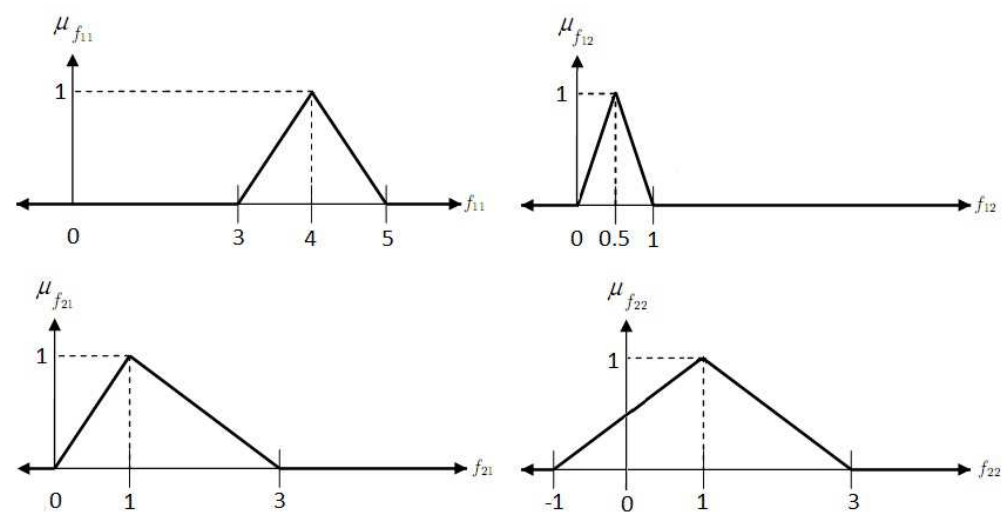

Figure 2: Triangular membership functions. 


$$
\begin{gathered}
\mu_{f_{11}}\left(f_{11}(x)\right)=\left\{\begin{array}{cl}
0 & \text { if } f_{11}\left(x_{1}, x_{2}\right) \geqslant c_{11} \\
\frac{c_{11}-f_{11}\left(x_{1}, x_{2}\right)}{c_{11}-b_{11}} & \text { if } b_{11} \leqslant f_{11}\left(x_{1}, x_{2}\right) \leqslant c_{11} \\
\frac{f_{11}\left(x_{1}, x_{2}\right)-a_{11}}{b_{11}-a_{11}} & \text { if } a_{11} \leqslant f_{11}\left(x_{1}, x_{2}\right) \leqslant b_{11}
\end{array}\right. \\
=\left\{\begin{array}{cll}
\frac{-2 x_{1}+9 x_{2}+3}{2 x_{2}+1} & \text { if } f_{11}\left(x_{1}, x_{2}\right) \leqslant a_{11} \\
\frac{2 x_{1}-5 x_{2}-1}{2 x_{2}+1} & \text { if } 4 \leqslant f_{11}\left(x_{1}, x_{2}\right) \leqslant 5 \\
0 & \text { if } 3 \leqslant f_{11}\left(x_{1}, x_{2}\right) \leqslant 4
\end{array}\right.
\end{gathered}
$$

In the same way, the other membership functions are formed as

$$
\begin{aligned}
& \mu_{f_{12}}\left(f_{12}(x)\right)=\left\{\begin{array}{cl}
1 & \text { if } f_{12}\left(x_{1}, x_{2}\right) \geqslant 1 \\
\frac{x_{2}+1}{0.5 x_{1}+0.5 x_{2}+0.5} & \text { if } 0.5 \leqslant f_{12}\left(x_{1}, x_{2}\right) \leqslant 1 \\
\frac{x_{1}}{0.5 x_{1}+0.5 x_{2}+0.5} & \text { if } 0 \leqslant f_{12}\left(x_{1}, x_{2}\right) \leqslant 0.5 \\
0 & \text { if } f_{12}\left(x_{1}, x_{2}\right) \leqslant 0
\end{array}\right. \\
& \mu_{f_{21}}\left(f_{21}(x)\right)=\left\{\begin{array}{cl}
0 & \text { if } f_{21}\left(x_{1}, x_{2}\right) \geqslant 3 \\
\frac{-6 x_{1}+x_{2}+13}{-2 x_{1}+2 x_{2}+8} & \text { if } 1 \leqslant f_{21}\left(x_{1}, x_{2}\right) \leqslant 3 \\
\frac{3 x_{1}+2 x_{2}-1}{-x_{1}+x_{2}+4} & \text { if } 0 \leqslant f_{21}\left(x_{1}, x_{2}\right) \leqslant 1 \\
0 & \text { if } f_{21}\left(x_{1}, x_{2}\right) \leqslant 0
\end{array}\right. \\
& \mu_{f_{22}}\left(f_{22}(x)\right)=\left\{\begin{array}{cll}
0 & \text { if } f_{22}\left(x_{1}, x_{2}\right) \geqslant 3 \\
\frac{4 x_{1}-x_{2}+12}{2 x_{1}+10} & \text { if } 1 \leqslant f_{22}\left(x_{1}, x_{2}\right) \leqslant 3 \\
\frac{x_{2}+8}{2 x_{1}+10} & \text { if }-1 \leqslant f_{22}\left(x_{1}, x_{2}\right) \leqslant 1 \\
0 & \text { if } f_{22}\left(x_{1}, x_{2}\right) \leqslant-1
\end{array}\right.
\end{aligned}
$$

If

$$
\mu_{f_{11}}\left(f_{11}(x)\right)=\max \left(\min \left(\frac{-2 x_{1}+9 x_{2}+3}{2 x_{2}+1}, \frac{2 x_{1}-5 x_{2}-1}{2 x_{2}+1}\right), 0\right)
$$

and

$$
\mu_{f_{12}}\left(f_{12}(x)\right)=\max \left(\min \left(\frac{x_{2}+1}{0.5 x_{1}+0.5 x_{2}+0.5}, \frac{x_{1}}{0.5 x_{1}+0.5 x_{2}+0.5}\right), 0\right)
$$


and

$$
\mu_{f_{21}}\left(f_{21}(x)\right)=\max \left(\min \left(\frac{-6 x_{1}+x_{2}+13}{-2 x_{1}+2 x_{2}+8}, \frac{3 x_{1}+2 x_{2}-1}{-x_{1}+x_{2}+4}\right), 0\right)
$$

and

$$
\mu_{f_{22}}\left(f_{22}(x)\right)=\max \left(\min \left(\frac{4 x_{1}-x_{2}+12}{2 x_{1}+10}, \frac{x_{2}+8}{2 x_{1}+10}\right), 0\right)
$$

Then the following results are obtained $\mu_{f_{11}}^{*}\left(f_{11}(1,0)\right), \mu_{f_{12}}^{*}\left(f_{12}((0,4)), \mu_{f_{21}}^{*}\left(f_{21}((1,0))\right.\right.$ and $\mu_{f_{22}}^{*}\left(f_{22}((0,4))\right.$. Now as before the membership functions are transformed by using first-order Taylor polynomial series.

$$
\begin{gathered}
\mu_{f_{11}}\left(f_{11}(x)\right) \cong \widehat{\mu_{f_{11}}}\left(f_{11}(x)\right)=\mu_{f_{11}}\left(f_{11}(1,0)\right)+\left(\left(x_{1}-1\right) \times \frac{\partial}{\partial x_{1}}+\right. \\
\left.\left(x_{2}-0\right) \times \frac{\partial}{\partial x_{2}}\right) \mu_{f_{11}}\left(f_{11}(1,0)\right)=-1+2 x_{1}-7 x_{2}
\end{gathered}
$$

In the same way, the other membership functions are transformed on using first-order Taylor polynomial series as follows:

$$
\begin{aligned}
& \mu_{f_{12}}\left(f_{12}(x)\right)=0.48+0.32 x_{1}-0.24 x_{2} \\
& \mu_{f_{21}}\left(f_{21}(x)\right)=1.78-0.61 x_{1}-0.22 x_{2} \\
& \mu_{f_{22}}\left(f_{22}(x)\right)=0.8-.24 x_{1}+0.1 x_{2}
\end{aligned}
$$

Adding (28) and (29), we get $\mathrm{P}(\mathrm{x})$,

$$
P(x)=\mu_{f_{11}}\left(f_{11}(x)\right)+\mu_{f_{12}}\left(f_{12}(x)\right)=-0.52+2.32 x_{1}-7.24 x_{2}
$$

After applying the Kuhn-Tucker conditions to the lower level of the objectives problem, a new auxiliary problem as below is to be solved

$$
\begin{array}{cl}
\operatorname{Max} & \quad P(x)=-0.52+2.32 x_{1}-7.24 x_{2} \\
\text { s.t } & \\
& x_{1}+x_{2}+u_{1}=4 \\
& x_{1}-2 x_{2}+u_{2}=1 \\
& 3 x_{1}+x_{2}-u_{3}=3 \\
& w_{1}-2 w_{2}-w_{3}-\nu=-0.22+0.1 \\
& w_{i} \leqslant M \eta_{i}, \quad u_{i} \leqslant M\left(1-\eta_{i}\right) \\
& x_{2} \leqslant M \xi, \quad \nu \leqslant M(1-\xi) \\
& \eta_{i}, \quad \xi \in\{0,1\}, \\
& x_{1}, \quad x_{2}, w_{i}, \quad u_{i}, \quad \nu \geqslant 0 \quad i=1, \ldots, 3
\end{array}
$$

The problem with $\mathrm{M}=1000$ is solved and the solution is obtained as follows:

$$
x_{1}^{*}=1, \quad x_{2}^{*}=0,
$$




$$
f_{11}\left(x_{1}, x_{2}\right)=4, f_{12}\left(x_{1}, x_{2}\right)=0.5, f_{21}\left(x_{1}, x_{2}\right)=0.67, f_{22}\left(x_{1}, x_{2}\right)=0.33
$$

and the membership values are

$$
\mu_{f_{11}}\left(f_{11}\right)=1, \quad \mu_{f_{12}}\left(f_{12}\right)=1, \quad \mu_{f_{21}}\left(f_{21}\right)=0.67, \quad \mu_{f_{22}}\left(f_{22}\right)=0.67
$$

The membership function values indicate that the level $f_{11}$ is satisfied $67 \%$, while $f_{12}$ is satisfied $100 \%$ accordingly $f_{21}, f_{22}$ are satisfiing with $0.67 \%$ with the solution of $x_{1}^{*}=1$ and $x_{2}^{*}=0$, The result indicates that the proposed solution method is very simple, efficient and robust. Finally in the same fashion the membership functions are considered to be trapezoidal (see Fig.3) which dependeds on four scalar parameters (a, b, c, d). Let $f_{11}$ be depends on four scalar parameters $(2,3,4,5), f_{12}$ on $(-1,0,1,3), f_{21}$ depends on four scalar parameters $(0,1,3,5)$ and $f_{22}$ be depends on $(0,1,2,4)$, as given by $f_{11}\left(x_{1}, x_{2} ; 2,3,4,5\right), f_{12}\left(x_{1}, x_{2} ;-1,0,1,3\right), f_{21}\left(x_{1}, x_{2} ; 0,1,3,5\right)$ and $f_{22}\left(x_{1}, x_{2} ; 0,1,2,4\right)$. The membership functions of the levels are obtained as follows:
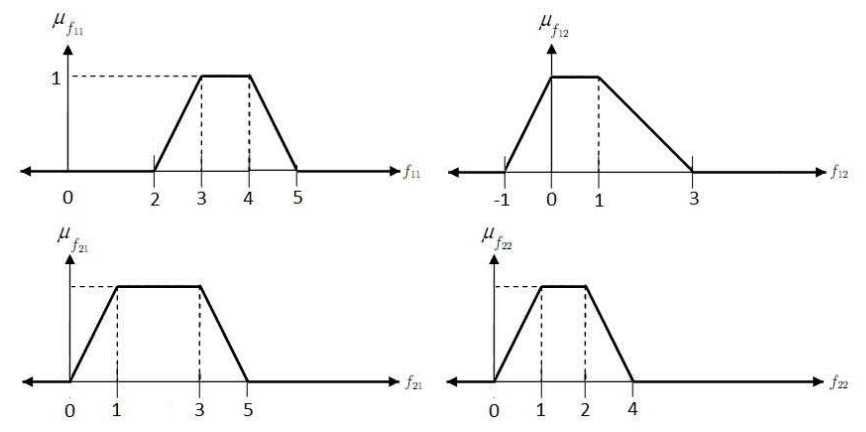

Figure 3: Trapezoidal membership functions

$$
\mu_{f_{11}}\left(f_{11}\right)=\left\{\begin{array}{clc}
0 & \text { if } f_{11}\left(x_{1}, x_{2}\right) \geqslant d_{11} \\
\frac{d_{11}-f_{11}\left(x_{1}, x_{2}\right)}{d_{11}-c_{11}} & \text { if } c_{11} \leqslant f_{11}\left(x_{1}, x_{2}\right) \leqslant d_{11} \\
1 & \text { if } b_{11} \leqslant f_{11}\left(x_{1}, x_{2}\right) \leqslant c_{11} \\
\frac{f_{11}\left(x_{1}, x_{2}\right)-a_{11}}{b_{11}-a_{11}} & \text { if } a_{11} \leqslant f_{11}\left(x_{1}, x_{2}\right) \leqslant b_{11} \\
0 & \text { if } f_{11}\left(x_{1}, x_{2}\right) \leqslant a_{11}
\end{array}\right.
$$




$$
\mu_{f_{11}}\left(f_{11}\right)=\left\{\begin{array}{cl}
0 & \text { if } f_{11}\left(x_{1}, x_{2}\right) \geqslant 5 \\
\frac{-2 x_{1}+9 x_{2}+3}{2 x_{2}+1} & \text { if } 4 \leqslant f_{11}\left(x_{1}, x_{2}\right) \leqslant 5 \\
1 & \text { if } 3 \leqslant f_{11}\left(x_{1}, x_{2}\right) \leqslant 4 \\
\frac{2 x_{1}-3 x_{2}}{2 x_{2}+1} & \text { if } 2 \leqslant f_{11}\left(x_{1}, x_{2}\right) \leqslant 3 \\
0 & \text { if } f_{11}\left(x_{1}, x_{2}\right) \leqslant 2
\end{array}\right.
$$

In the same way, the other membership functions are formed as

$$
\begin{gathered}
\mu_{f_{12}}\left(f_{12}\right)=\left\{\begin{array}{cl}
0 & \text { if } f_{12}\left(x_{1}, x_{2}\right) \geqslant 3 \\
\frac{2 x_{1}+3 x_{2}+3}{2 x_{1}+2 x_{2}+2} & \text { if } 1 \leqslant f_{12}\left(x_{1}, x_{2}\right) \leqslant 3 \\
1 & \text { if } 0 \leqslant f_{12}\left(x_{1}, x_{2}\right) \leqslant 1 \\
\frac{2 x_{1}+x_{2}+1}{x_{1}+x_{2}+1} & \text { if }-1 \leqslant f_{12}\left(x_{1}, x_{2}\right) \leqslant 0 \\
0 & \text { if } f_{12}\left(x_{1}, x_{2}\right) \leqslant-1
\end{array}\right. \\
\mu_{f_{21}}\left(f_{21}\right)=\left\{\begin{array}{cl}
\frac{-8 x_{1}+3 x_{2}+21}{-2 x_{1}+2 x_{2}+8} & \text { if } 3 \leqslant f_{21}\left(x_{1}, x_{2}\right) \leqslant 5 \\
1 & \text { if } 1 \leqslant f_{21}\left(x_{1}, x_{2}\right) \leqslant 3 \\
\frac{3 x_{1}+2 x_{2}-1}{-x_{1}+x_{2}+4} & \text { if } 0 \leqslant f_{21}\left(x_{1}, x_{2}\right) \leqslant 1 \\
0 & \text { if } f_{21}\left(x_{1}, x_{2}\right) \leqslant 0
\end{array}\right. \\
\mu_{f_{22}}\left(f_{22}\right)=\left\{\begin{array}{cll}
\frac{-x_{1}+x_{2}+3}{x_{1}+5} & \text { if } 0 \leqslant f_{22}\left(x_{1}, x_{2}\right) \leqslant 1 \\
0 & \text { if } f_{22}\left(x_{1}, x_{2}\right) \leqslant 0 \\
\frac{5 x_{1}-x_{2}+17}{2 x_{1}+10} & \text { if } 2 \leqslant f_{22}\left(x_{1}, x_{2}\right) \leqslant 4 \\
\frac{-1}{0} & \text { if } 1 \leqslant f_{22}\left(x_{1}, x_{2}\right) \leqslant 2
\end{array}\right.
\end{gathered}
$$

If

$$
\mu_{f_{11}}\left(f_{11}(x)\right)=\max \left(\min \left(\frac{-2 x_{1}+9 x_{2}+3}{2 x_{2}+1}, 1, \frac{2 x_{1}-3 x_{2}}{2 x_{2}+1}\right), 0\right)
$$

and

$$
\mu_{f_{12}}\left(f_{12}(x)\right)=\max \left(\min \left(\frac{2 x_{1}+3 x_{2}+3}{2 x_{1}+2 x_{2}+2}, 1, \frac{2 x_{1}+x_{2}+1}{x_{1}+x_{2}+1}\right), 0\right)
$$


and

$\mu_{f_{21}}\left(f_{21}(x)\right)=\max \left(\min \left(\frac{-8 x_{1}+3 x_{2}+21}{-2 x_{1}+2 x_{2}+8}, 1, \frac{3 x_{1}+2 x_{2}-1}{-x_{1}+x_{2}+4}\right), 0\right)$

and

$$
\mu_{f_{22}}\left(f_{22}(x)\right)=\max \left(\min \left(\frac{5 x_{1}-x_{2}+17}{2 x_{1}+10}, 1, \frac{-x_{1}+x_{2}+3}{x_{1}+5}\right), 0\right)
$$

then $\mu_{f_{11}}^{*}\left(f_{11}(1,0), \mu_{f_{12}}^{*}\left(f_{12}(3,1), \mu_{f_{21}}^{*}\left(f_{21}(1,0)\right.\right.\right.$ and $\mu_{f_{22}}^{*}\left(f_{22}(0,4)\right.$ is obtained. Now, the membership functions are transformed by using first-order Taylor polynomial series.

In the same way, the other membership functions are transformed by using first-order Taylor polynomial series, in which we obtain.

$$
\begin{aligned}
& \mu_{f_{12}}\left(f_{12}(x)\right)=1.48+0.08 x_{1}-0.12 x_{2} \\
& \mu_{f_{21}}\left(f_{21}(x)\right)=2.77-0.61 x_{1}-0.22 x_{2} \\
& \mu_{f_{22}}\left(f_{22}(x)\right)=0.6-0.32 x_{1}+0.2 x_{2}
\end{aligned}
$$

The $\mathrm{P}(\mathrm{x})$ is obtained by adding (42) and (43) as follows:

$$
P(x)=\mu_{f_{11}}\left(f_{11}(x)\right)+\mu_{f_{12}}\left(f_{12}(x)\right)=1.48+2.08 x_{1}-7.12 x_{2}
$$

After applying the Kuhn-Tucker conditions to the lower level of the objectives problem, a new auxiliary problem is to be solved.

$$
\begin{array}{cl}
\text { Max } & \quad P(x)=1.48+2.08 x_{1}-7.12 x_{2} \\
\text { s.t } & \\
& x_{1}+x_{2}+u_{1}=4 \\
& x_{1}-2 x_{2}+u_{2}=1 \\
& 3 x_{1}+x_{2}-u_{3}=3 \\
& w_{1}-2 w_{2}-w_{3}-\nu=-0.22+0.2 \\
& w_{i} \leqslant M \eta_{i}, \quad u_{i} \leqslant M\left(1-\eta_{i}\right) \\
& x_{2} \leqslant M \xi, \quad \nu \leqslant M(1-\xi) \\
& \eta_{i}, \quad \xi \in\{0,1\}, \quad{ }^{2}, \quad x_{i}, \quad w_{i} \geqslant 0 \quad i=1, \ldots, 3
\end{array}
$$

The problem with $M=1000$, is solved and the solution is obtained as follows:

$$
\begin{gathered}
x_{1}^{*}=1, \quad x_{2}^{*}=0, \\
f_{11}\left(x_{1}, x_{2}\right)=4, f_{12}\left(x_{1}, x_{2}\right)=0.5, f_{21}\left(x_{1}, x_{2}\right)=0.67, f_{22}\left(x_{1}, x_{2}\right)=0.33
\end{gathered}
$$

with the membership values as

$$
\mu_{f_{11}}\left(f_{11}\right)=1, \quad \mu_{f_{12}}\left(f_{12}\right)=1, \quad \mu_{f_{21}}\left(f_{21}\right)=0.67, \quad \mu_{f_{22}}\left(f_{22}\right)=0.33
$$

The membership function values indicate that the levels $f_{11} \& f_{12}$ are satisfied $100 \%$ while $f_{21}$ is satisfied with $0.67 \%$ and $f_{22}$ with $0.33 \%$ with the optimal solution of $x_{1}^{*}=3$ and $x_{2}^{*}=1$. 


\section{Conclusion}

In this paper, for solving FLFBL-MOP problems a powerful and robust method which is based on Taylor series and Kuhn-Tucker conditions is proposed. Membership functions associated with each level of each objective is transformed by using Taylor series. Actually, the FLFBL-MOP problem is reduced to an equivalent single objective linear programming problem by using the first-order Taylor polynomial series and Kuhn-Tucker conditions and then the proposed solution method is applied to numerical example to test the effect on the performance. It is obvious that the results show that the proposed method is more effective in reducing the complexity in computation for solving problems.

\section{References}

[1] O. Ben-Ayed, Bilevel linear programming, Computers and Operations Research20 (1993) 485501.

[2] M. Sakawa, I. Nishizaki, Interactive fuzzy programming for decentralized two-level linear programming problems, Fuzzy Sets and Systems125 (2002) 301315 .

[3] M. Sakawa, I. Nishizaki, Interactive fuzzy programming for two-level linear fractional programming problems, Fuzzy Sets and Systems119 (2001) $31-40$.

[4] M. Saraj, N. Safaei, Solving Bi-Level Programming Problems on Using Global Criterion Method with an Interval Approach, Applied Mathematical Sciences 6 (2012) 1135-1141.

[5] M. A. Abo-Sinna, A bilevel non-linear multi-objective decision making under fuzziness, Journal of Operational Research Society of India38 (2001) 484-495.

[6] M. S. Osman, M. A. Abo-Sinna, A. H. Amer, O. E. Emam, A multilevel non-linear multiobjective decision-making under fuzziness, Applied Mathematics and Computation153 (2004) 239252.

[7] H. J. Zimmermann, Fuzzy programming and linear programming with several objective functions, Fuzzy Sets and Systems1 (1978) 45-55.

[8] Y. J. Lai, Hierarchical optimization: A satisfactory solution, Fuzzy Sets and Systems77 (1996)321-335. 
[9] B. B. Pal, B. N. Moitra, A fuzzy goal programming procedure for solving quadratic bilevel programming problems, International Journal of Intelligent Systems18 (2003) 529-540.

[10] H. S. Shih, Y. J. Lai, E. S. Lee, Fuzzy approach for multi-level programming problems, Computers and Operations Research 23 (1996) 73-91.

[11] S. Sinha, Fuzzy programming approach to multi-level programming problems, Fuzzy Sets and Systems 136 (2003) 189-202.

[12] I. A. Baky, Fuzzy goal programming algorithm for solving decentralized bi-level multi-objective programming problems, Fuzzy Sets and Systems 160 (2009) 2701-2713. 